\title{
Common Fixed Points of Single and Multivalued Maps in Fuzzy Metric Spaces
}

\author{
Palaniappan Vijayaraju ${ }^{1}$, Zahurullah Mohamed Ibrahim Sajath ${ }^{2}$ \\ ${ }^{1}$ Department of Mathematics, Anna University, Chennai, India \\ ${ }^{2}$ Department of Information Technology, Higher College of Technology, Muscat, Sultanate of Oman \\ E-mail:vijay@annauniv.edu,misajath@yahoo.com \\ Received February 24, 2011; revised March 25, 2011; accepted March 29, 2011
}

\begin{abstract}
In this paper we introduce the notion of common property (EA) in fuzzy metric spaces. Further we prove some common fixed points theorems for hybrid pair of single and multivalued maps under hybrid contractive conditions. Our results extend previous ones in fuzzy metric spaces.
\end{abstract}

Keywords: Fuzzy Metric Space, Common Fixed Point, Coincidence Point

\section{Introduction}

In 1965 Zadeh [1] introduced the theory of fuzzy sets. Many authors introduced the notion of fuzzy metric space in different ways. George and Veeramani [2] modified the concept of fuzzy metric space introduced by Kramosil and Michalek [3] and defined Haussdorf topology in fuzzy metric space. Several authors [4-11] studied and developed the concept in different directions and proved fixed point theorems in fuzzy metric spaces.

In 1986 Jungck [12] introduced the concept of compatible mappings and utilized it to improve and generalize the commutativity conditions employed in common fixed point theorems. This induced interest in non-compatible mappings initiated by Pant [13]. Recently Aamri and Moutawakil [14] and Liu et al. [15] respectively defined the property (E.A) and the common property (E.A) as a generalization of non-compatibility and proved some common fixed point theorems in metric spaces. The aim of this paper is to define the common property (E.A) in the settings of fuzzy metric space and utilize the same to obtain some common fixed point theorems in fuzzy metric spaces.

We begin with some definitions and preliminary concepts.

\section{Preliminaries}

Definition 2.1. [16] A binary operation *:[0,1] $\times[0,1]$ $\rightarrow[0,1]$ is called a continuous $t$-norm if $([0,1], *)$ is an abelian toplological monoid with unit 1 such that $a^{*} b \leq c * d$ whenever $a \leq c$ and $b \leq d$ for all $a, b, c, d \in[0,1]$.

Examples of $t$-norm are $a^{*} b=a b$ and $a^{*} b=\min \{a, b\}$.

Definition 2.2. [3]. A triplet $(X, M, *)$ is said to be a fuzzy metric space if $X$ is an arbitrary set, ${ }^{*}$ is a continuous $t$-norm, and $M$ is a fuzzy set on

$X^{2} \times[0, \infty)$ satisfying the following conditions: for all $x, y, z \in X$ and all $s, t>0$,

1) $M(x, y, 0)=0$

2) $M(x, y, t)=1$ for all $t>0$ if and only if $x=y$;

3) $M(x, y, t)=M(y, x, t)$;

4) $M(x, y, t) * M(y, z, s) \leq M(x, z, t+s)$;

5) $M(x, y,):.[0, \infty) \rightarrow[0,1]$ is left continuous;

6) $\lim _{t \rightarrow \infty} M(x, y, t)=1$.

$M$ is called fuzzy metric on $X$. The functions $M(x, y, t)$ denote the degree of nearness between $x$ and $y$ with respect to $t$ respectively.

Definition 2.3. Let $(X, M, *)$ be a fuzzy metric space.

A sequence $\left\{x_{n}\right\}$ in $X$ is called Cauchy sequence if and only if $\lim _{n \rightarrow \infty} M\left(x_{n+p}, x_{n}, t\right)=1$ for each $p>0$, $t>0$.

A sequence $\left\{x_{n}\right\}$ in $X$ is converging to $x$ in $X$ if and only if $\lim _{n \rightarrow \infty} M\left(x_{n}, x, t\right)=1$.

A fuzzy metric space $\left(X, M,{ }^{*}\right)$ is said to be complete if and only if every Cauchy sequence in $X$ is convergent in $X$.

Definition 2.4. [8] Let $\mathcal{C B}(X)$ denote the set of all nonempty closed bounded subsets of $X$. Then for every $A, B, C \in \mathcal{C B}(X)$ and $t>0$, 


$$
\begin{aligned}
& M_{\nabla}(A, B, t)= \\
& \min \left\{\min _{a \in A} M^{\nabla}(a, B, t), \min _{b \in B} M^{\nabla}(A, b, t)\right\}
\end{aligned}
$$

where $M^{\nabla}(C, y, t)=\max \{M(z, y, t): z \in C\}$.

Remark 2.5. Obviously $M_{\nabla}(A, B, t) \leq M^{\nabla}(a, B, t)$ whenever $a \in A$ and $M_{\nabla}(A, B, t)=1$ if and only if $A=B$.

Definition 2.6. [9] Two mappings $f$ and $g$ are compatible if and only if $\lim _{n \rightarrow \infty} M\left(f g\left(x_{n}\right), g f\left(x_{n}\right), t\right)=1$ for all $t>0$, whenever $\left\{x_{n}\right\}$ is a sequence in $X$ such that $\lim _{n \rightarrow \infty} f\left(x_{n}\right)=\lim _{n \rightarrow \infty} g\left(x_{n}\right)=x_{o} \in X$.

Lemma 2.7. Let $f, g$ be two compatible mappings on $X$. If $f(x)=g(x)$ for some $x$ in $X$, then $f g(x)=g f(x)$.

Definition 2.8. [10,11] Maps $f, g: X \mapsto X$ are said to satisfy the property (EA) if there exists a sequence $\left\{x_{n}\right\} \in X$ such that $\lim _{n \rightarrow \infty} f x_{n}=\lim _{n \rightarrow \infty} g x_{n}=t \in X$.

Definition 2.9. [11] A point in $X$ is a coincidence point (fixed point) of $f$ and $T$ if

$$
f(x)=T(x)(T(x)=f(x)=x) .
$$

Definition 2.10. [11] A point $x$ in $X$ is a coincidence point of $f: X \mapsto X$ and $T: X \mapsto \mathcal{C B}(X)$ if $f(x) \in T(x)$. We denote the set of all coincidence points of $f$ and $T$ by $C(f, T)$.

Definition 2.11. [16] Maps $f: X \mapsto X$ and $T: X \mapsto \mathcal{C B}(X)$ are weakly compatible if they commute at their coincidence points, that is, if $f T x=T f x$ whenever $f x \in T x$.

Definition 2.12. [18] Maps $f: X \mapsto X$ and $T: X \mapsto \mathcal{C B}(X)$ are said to be $(\mathcal{I T})$ commuting at $x \in X$ if $f T x \subset T f x$ whenever $f x \in T x$.

Definition 2.13. [19] Maps $f: X \mapsto X$ and $T: X \mapsto \mathcal{C B}(X)$ are said to satisfy the property (EA) if there exists a sequence $\left\{x_{n}\right\} \in X$, some $t \in X$ and $A \in \mathcal{C B}(X)$ such that $\lim _{n \rightarrow \infty} f x_{n}=t \in A=\lim _{n \rightarrow \infty} T x_{n}$.

Definition 2.14. [19] Let $T: X \mapsto \mathcal{C B}(X)$. The map $f: X \mapsto X$ is said to be $T$ weakly commuting at $x \in X$ if $f f x \in T f x$.

\section{Main Results}

We begin with the following definition.

Definition 3.1. [11] Let $\left(X, M,{ }^{*}\right)$ be a fuzzy metric space and $f, g . F, G: X \mapsto X$. The maps pair $(f, F)$ and $(g, G)$ are said to satisfy the common property (EA) if there exist two sequences $\left\{x_{n}\right\},\left\{y_{n}\right\} \in X$ and some $t$ in $X$ such that

$$
\begin{aligned}
& \lim _{n \rightarrow \infty} G y_{n}= \\
& \lim _{n \rightarrow \infty} F x_{n}=\lim _{n \rightarrow \infty} g y_{n}=\lim _{n \rightarrow \infty} f x_{n}=t \in X
\end{aligned} .
$$

Definition 3.2. Let $(X, M, *)$ be a fuzzy metric space, $f, g: X \mapsto X$ and $F, G: X \mapsto \mathcal{C B}(X)$. The maps pair $(f, F)$ and $(g, G)$ are said to satisfy the common property $(E A)$ if there exist two sequences $\left\{x_{n}\right\},\left\{y_{n}\right\} \in X$, some $t \in X$ and $A, B \in \mathcal{C B}(X)$ such that

$$
\lim _{n \rightarrow \infty} F x_{n}=A, \lim _{n \rightarrow \infty} G y_{n}=B, \lim _{n \rightarrow \infty} f x_{n}=\lim _{n \rightarrow \infty} g y_{n}=t \in A \cap B .
$$

Theorem 3.3. Let $f, g$ be two self maps of the fuzzy metric space $(X, M, *)$ and let $F, G$ be two maps from $X$ into $\mathcal{C B}(X)$ such that
1) $(f, F)$ and $(g, G)$ satisfy the common property (EA);

2) for all $x \neq y$ in $X$,

$$
M_{\nabla}\left(F x_{n}, G w, t\right)>\min \left\{M\left(f x_{n}, g w, t\right), \frac{M^{\nabla}(f x, F x, t)+M^{\nabla}(g y, G y, t)}{2}, \frac{M^{\nabla}(f x, G y, t)+M^{\nabla}(g y, F x, t)}{2}\right\}
$$

If $f X$ and $g X$ are closed in $X$, then

1) $f$ and $F$ have a coincidence point;

2) $g$ and $G$ have a coincidence point;

3) $f$ and $F$ have a common fixed point provided that $f$ is $F$ weakly commuting at $v$ and $f f v=f v$ for $v \in C(f, F)$;

4) $g$ and $G$ have a common fixed point provided that $g$ is $G$ weakly commuting at $v$ and $g g v=g v$ for $v \in C(g, G)$;

5) $f, F, g$ and $G$ have a common fixed point provided that both $(c)$ and $(d)$ are true.

$$
M_{\nabla}\left(F x_{n}, G w, t\right)>\min M\left\{\left(f x_{n}, g w, t\right), \frac{M^{\nabla}\left(f x_{n}, F x_{n}, t\right)+M^{\nabla}(g w, G w, t)}{2}, \frac{M^{\nabla}\left(f x_{n}, G w, t\right)+M^{\nabla}\left(g w, F x_{n}, t\right)}{2}\right\}
$$

Proof. Since $(f, F)$ and $(g, G)$ satisfy the common property $(E A)$, there exist two sequences $\left\{x_{n}\right\}$ and $\left\{y_{n}\right\}$ in $X, u \in X$ and $A, B \in \mathcal{C B}(X)$ such that $\lim _{n \rightarrow \infty} F x_{n}=A$ and $\lim _{n \rightarrow \infty} G y_{n}=B$,

$$
\lim _{n \rightarrow \infty} f x_{n}=\lim _{n \rightarrow \infty} g y_{n}=u \in A \cap B
$$

By virtue of $f(x)$ and $g(x)$ being closed, we have $u=f v$ and $u=g w$ for some $v, w \in X$. Now we shall show that $f v \in F v$ and $g w \in G w$. The condition (2) implies that 
Taking the limit as $n \rightarrow \infty$, we get

$$
M_{\nabla}(A, G w, t) \geq \min \left\{M(f v, g w, t), \frac{M^{\nabla}(f v, A, t)+M^{\nabla}(g w, G w, t)}{2}, \frac{M^{\nabla}(f v, G w, t)+M^{\nabla}(g w, A, t)}{2}\right\}
$$

Since $u=f v=g w$, we obtain

$$
M_{\nabla}(A, G w, t) \geq \min \left\{1, \frac{M^{\nabla}(u, A, t)+M^{\nabla}(u, G w, t)}{2}, \frac{M^{\nabla}(u, G w, t)+M^{\nabla}(u, A, t)}{2}\right\}
$$

That is, $M_{\nabla}(A, G w, t) \geq \frac{1+M^{\nabla}(u, G w, t)}{2}$, since $u \in A$.

$$
\Rightarrow 2 M_{\nabla}(A, G w, t) \geq\left\{1+M^{\nabla}(u, G w, t)\right\}
$$

But from (2.5), we have $M_{\nabla}(A, G w, t) \leq M^{\nabla}(u, G w, t)$

That is $2 M_{\nabla}(A, G w, t) \leq 2 M^{\nabla}(u, G w, t)$
Combining the inequalities (3) and (4) we get $1+M^{\nabla}(u, G w, t) \leq 2 M_{\nabla}(A, G w, t) \leq 2 M^{\nabla}(u, G w, t)$

This implies $1+M^{\nabla}(u, G w, t) \leq 2 M^{\nabla}(u, G w, t)$

$$
\Rightarrow M^{\nabla}(u, G w, t) \geq 1 \Rightarrow M^{\nabla}(u, G w, t)=1
$$

Hence $u=g w \in G w$

On the other hand by condition (2), we have

$$
M_{\nabla}\left(F v, G y_{n}, t\right)>\min \left\{M\left(f v, g y_{n}, t\right), \frac{M^{\nabla}(f v, F v, t)+M^{\nabla}\left(g y_{n}, G y_{n}, t\right)}{2}, \frac{M^{\nabla}\left(f v, G y_{n}, t\right)+M^{\nabla}\left(g y_{n}, F v, t\right)}{2}\right\}
$$

Taking limit as $n \rightarrow \infty$, we get

$$
M_{\nabla}(F v, B, t) \geq \min \left\{M(f v, g w, t), \frac{M^{\nabla}(f v, F v, t)+M^{\nabla}(g w, B, t)}{2}, \frac{M^{\nabla}(f v, B, t)+M^{\nabla}(g w, F v, t)}{2}\right\}
$$

That is,

$$
\begin{aligned}
& M_{\nabla}(F v, B, t) \\
& \geq \min \left\{1, \frac{M^{\nabla}(f v, F v, t)+1}{2}, \frac{1+M^{\nabla}(g w, F v, t)}{2}\right\}
\end{aligned}
$$

Similarly, we obtain $M^{\nabla}(f v, F v, t)=1$, which implies that $f v \in F v$. Thus $f$ and $F$ have a coincidence point $v, g$ and $G$ have a coincidence point $w$. This ends the proof of (a) and (b).

By virtue of condition (c), we get $f f v \in F f v$. Thus $u=f u \in F u$. This proves (c). Similarly (d) can be proved. Then (e) follows immediately.

Corollary 3.4. Let $f$ be a self-map of fuzzy metric space $(X, M, *)$ and let $F$ be a map from $X$ into $\mathcal{C B}(X)$ such that

1) $(f, F)$ satisfies the property $(E A)$;

2) for all $x \neq y$ in $X$,

$$
M_{\nabla}(F x, F y, t)>\min \left\{M(f x, f y, t), \frac{M^{\nabla}(f x, F x, t)+M^{\nabla}(f y, F y, t)}{2}, \frac{M^{\nabla}(f x, F y, t)+M^{\nabla}(f y, F x, t)}{2}\right\}
$$

If $f X$ is closed subset of $X$, then

1) $f$ and $F$ have a coincidence point;

2) $f$ and $F$ have a common fixed point provided that $f$ is $F$ weakly commuting at $v$ and $f f v=f v$, for $v \in C(f, F)$.

Proof. Let $F=G$ and $f=g$, then the result follows.

$$
M_{\nabla}(F x, G y, t)>\min \left\{M(f x, f y, t), \frac{M^{\nabla}(f x, F x, t)+M^{\nabla}(f y, G y, t)}{2}, \frac{M^{\nabla}(f x, G y, t)+M^{\nabla}(f y, F x, t)}{2}\right\}
$$

Corollary 3.5. Let $f$ be a self-map of the fuzzy metric space $(X, M, *)$ and let $F$ and $G$ be two maps from $X$ into $\mathcal{C B}(X)$ such that

1) $(f, F)$ and $(f, G)$ satisfy the common property (EA) ;

2) for all $x \neq y$ in $X$. 
If $f X$ is closed subset of $X$, then

1) $f, G$ and $F$ have a coincidence point;

2) $f, G$ and $F$ have a common fixed point provided that $f$ is both $F$ weakly commuting and $G$ weakly commuting at $v$ and $f f v=f v$, for $v \in C(f, F)$.

Proof. Let $f=g$, then the result follows.
If both $F$ and $G$ are single valued maps in theorem 3.3 , then we have the following corollary.

Corollary 3.6. Let $f, g, F$ and $G$ be four selfmaps of the fuzzy metric space $(X, M, *)$ such that

1) $(f, F)$ and $(f, G)$ satisfy the common property $(E A)$;

2) for all $x \neq y$ in $X$,

$$
M_{\nabla}(F x, G y, t)>\min \left\{M(f x, g y, t), \frac{M^{\nabla}(f x, F x, t)+M^{\nabla}(g y, G y, t)}{2}, \frac{M^{\nabla}(f x, G y, t)+M^{\nabla}(g y, F x, t)}{2}\right\}
$$

If $f X$ and $g X$ are closed subsets of $X$, then

1) $f$ and $F$ have a coincidence point;

2) $g$ and $G$ have a coincidence point;

3) $f$ and $F$ have a common fixed point provided that $f$ is $F$ weakly commuting at $v$ and $f f v=f v$ for $v \in C(f, F)$;

4) $g$ and $G$ have a common fixed point provided that $g$ is $G$ weakly commuting at $v$ and $g g v=g v$ for $v \in C(g, G)$;

5) $f, F, g$ and $G$ have a common fixed point provided that both (c) and (d) are true.
The next theorem involves a continuous function $\varphi:[0,1] \mapsto[0,1]$ satisfying the following conditions:

$\left(A_{1}\right) \varphi$ is nonincreasing on $[0,1]$,

$\left(A_{2}\right) \varphi(t)>t$ for each $t \in(0,1)$.

Theorem 3.7. Let $f, g$ be two self maps of the fuzzy metric space $(X, M, *)$ and let $F, G$ be two maps from $X$ into $\mathcal{C B}(X)$ such that

1) $(f, F)$ and $(g, G)$ satisfy the common property $(E A)$

2) for all $x \neq y$ in $X$,

$$
M_{\nabla}(F x, G y, t) \geq \varphi\left(\min \left\{M(f x, g y, t), M^{\nabla}(f x, F x, t), M^{\nabla}(g y, G y, t), M^{\nabla}(f x, G y, t), M^{\nabla}(g y, F x, t)\right\}\right)
$$

If $f X$ and $g X$ are closed in $X$, then

1) $f$ and $F$ have a coincidence point;

2) $g$ and $G$ have a coincidence point;

3) $f$ and $F$ have a common fixed point provided that $f$ is $F$ weakly commuting at $v$ and $f f v=f v$ for $v \in C(f, F)$;

4) $g$ and $G$ have a common fixed point provided that $g$ is $G$ weakly commuting at $v$ and $g g v=g v$ for $v \in C(g, G)$;

5) $f, F, g$ and $G$ have a common fixed point provided that both $(c)$ and $(d)$ are true.
Proof. Since $(f, F)$ and $(g, G)$ satisfy the common property $(E A)$, there exist two sequences $\left\{x_{n}\right\}$ and $\left\{y_{n}\right\}$ in $X, u \in X$ and $A, B \in \mathcal{C B}(X)$ such that $\lim _{n \rightarrow \infty} F X_{n}=A$ and $\lim _{n \rightarrow \infty} G y_{n}=B$,

$$
\lim _{n \rightarrow \infty} f x_{n}=\lim _{n \rightarrow \infty} g y_{n}=u \in A \cap B .
$$

By virtue of $f(x)$ and $g(x)$ being closed, we have $u=f v$ and $u=g w$ for some $v, w \in X$. we claim that $f v \in F v$ and $g w \in G w$. Indeed condition (2) implies that

$$
M_{\nabla}\left(F x_{n}, G w, t\right) \geq \varphi\left(\min \left\{M\left(f x_{n}, g w, t\right), M^{\nabla}\left(f x_{n}, F x_{n}, t\right), M^{\nabla}(g w, G w, t), M^{\nabla}\left(f x_{n}, G w, t\right), M^{\nabla}\left(g w, F x_{n}, t\right)\right\}\right)
$$

Taking limit as $n \rightarrow \infty$, we obtain

$$
M_{\nabla}(A, G w, t) \geq \varphi\left(\min \left\{M(f v, g w, t), M^{\nabla}(f v, A, t), M^{\nabla}(g w, G w, t), M^{\nabla}(f v, G w, t), M^{\nabla}(g w, A, t)\right\}\right)
$$

Since $f v=g w \in A \cap B$, we get

$$
M(f v, g w, t)=M(f v, A, t)=M^{\nabla}(g w, A, t)=1 .
$$

Therefore

$$
\begin{gathered}
M_{\nabla}(A, G w, t) \geq \varphi\left(M^{\nabla}(f v, G w, t)\right)>M^{\nabla}(f v, G w, t) . \quad \text { On the other hand by condition (2) again, we have } \\
M_{\nabla}\left(F v, G y_{n}, t\right) \geq \varphi\left(\min \left\{M\left(f v, g y_{n}, t\right), M^{\nabla}(f v, F v, t), M^{\nabla}\left(g y_{n}, G y_{n}, t\right), M^{\nabla}\left(f v, G y_{n}, t\right), M^{\nabla}\left(g y_{n}, F v, t\right)\right\}\right)
\end{gathered}
$$

That is $M_{\nabla}(A, G w, t)>M^{\nabla}(f v, G w, t)$.

This contradicts 2.5 and hence $M_{\nabla}(A, G w, t)=1$. This implies that $A=G w$. Therefore $f v=g w \in G w$. 
Similarly, taking limit as $n \rightarrow \infty$, we obtain

$$
M_{\nabla}(F v, B, t)=1
$$

Thus, we get $F v=B$.

Since $f v=g w \in B, f v \in F v$.

Thus $f$ and $F$ have a coincidence point $v, g$ and $G$ have a coincidence point $w$. This ends the proof of part (a) and part (b). The rest of proof is similar to the argument of theorem 2.3.

\section{References}

[1] L. A. Zadeh, "Fuzzy Sets," Information and Control, Vol. 8, No. 3, 1965, pp. 338-353. doi:10.1016/S0019-9958(65)90241-X

[2] A. George and P. Veeramani, "On Some Results in Fuzzy Metric Spaces,” Fuzzy Sets and Systems, Vol. 64, No. 3, 1994, pp. 395-399. doi:10.1016/0165-0114(94)90162-7

[3] I. Karmosil and J. Michalek, "Fuzzy Metric Statistical Metric Spaces,” Kybernetica, Vol. 11, No.5, 1975, pp. 336-344.

[4] Z. K. Deng, “Fuzzy Pseudo-Metric Spaces,” Journal of Mathematical Analysis and Applications, Vol. 86, No. 1, 1982, pp. 74-95. doi:10.1016/0022-247X(82)90255-4

[5] M. Grabiec, "Fixed Points in Fuzzy Metric Spaces," Fuzzy Sets and Systems, Vol. 27, No. 3, 1988, pp. 385-389. doi:10.1016/0165-0114(88)90064-4

[6] O. Kaleva and S. Seikkala, "On Fuzzy Metric Spaces,” Fuzzy Sets and Systems, Vol. 12, No. 3, 1984, pp. 215-229. doi:10.1016/0165-0114(84)90069-1

[7] S. Sharma, "Common Fixed Point Theorems in Fuzzy Metric Spaces,” Fuzzy Sets and Systems, Vol. 127, No. 3, 2002, pp. 345-352. doi:10.1016/S0165-0114(01)00112-9

[8] I. Kubiaczyk and S. Sharma, "Common Coincidence Point in Fuzzy Metric Spaces,” Journal of Fuzzy Mathematics, Vol. 11, No. 1, 2003, pp. 1-5.

[9] B. Singh and M. S. Chauhan, "Common Fixed Points of Compatible Maps in Fuzzy Metric Spaces,” Fuzzy Sets and Systems, Vol. 115, No. 3, 2000, pp. 471-475. doi:10.1016/S0165-0114(98)00099-2

[10] P. Vijayaraju and Z. M. I. Sajath, "Some Common Fixed Point Theorems in Fuzzy Metric Spaces,” International Journal of Mathematical Analysis, Vol. 3, No. 15, 2009, pp. 701-710.

[11] M. Abbas, I. Altun and D. Gopal, "Comon Fixed Point Theorems for Non Compatible Mappings in Fuzzy Metric Spaces," Bulletin of Mathematical Analysis and Applications, Vol. 1, No. 2, 2009, pp. 47-56.

[12] G. Jungk, "Compatible Mappings and Common Fixed Points,” International Journal of Mathematical Analysis, Vol. 9, No. 4, 1986, pp. 771-779.

[13] R. P. Pant, "Common Fixed Points of Non Commutative Mappings," Journal of Mathematical Analysis and Applications, Vol. 188, No. 1, 1994, pp. 436-440. doi:10.1006/jmaa.1994.1437

[14] A. Aamri and D. E. Moutawkil, "Some New Common Fixed Point Theorems under Strict Contractive Conditions," Journal of Mathematical Analysis and Applications, Vol. 270, No. 1, 2002, pp. 181-188. doi:10.1016/S0022-247X(02)00059-8

[15] Y. Liu, J. Wu and Z. Li, "Common Fixed Points of Single Valued and Multivalued Maps,” International Journal of Mathematics and Mathematical Sciences, Vol. 2005, No. 19, 2005, pp. 3045-3055. doi:10.1155/IJMMS.2005.3045

[16] B. Schweizer and A. Sklar, "Statistical Metric Spaces," Pacific Journal of Mathematics, Vol. 10, 1960, pp. 331334.

[17] B. E. Rhoades and G. Jungck, "Fixed Points for Set Valued Function without Continuity," Indian Journal of Pure and Applied Mathematics, Vol. 29, No. 3, 1998, pp. 227-238.

[18] S. L. Singh and S. N. Mishra, "Coincidences and Fixed Points of Nonself Hybrid Contractions," Journal of Mathematical Analysis and Applications, Vol. 256, No. 2, 2001, pp. 486-497. doi:10.1006/jmaa.2000.7301

[19] T. Kamran, "Coincidence and Fixed Points for Hybrid Strict Contractions,” Journal of Mathematical Analysis and Applications, Vol. 299, No. 1, 2004, pp. 23-24. 